\title{
Von den Gammafunctionen und einer besonderen Art unendlicher Producte.
}

(Von Herrn G. Bauer zu München.)

Gau/s bemerkt in seiner Abhandlung "Disquisitiones generales circa seriem infinitam etc." dafs die Stirlingsche Formel für $\log (1.2 .3 \ldots a)$ überhaupt für $\log I^{\prime}(a+1)$ gültig sei, wenn $a$ irgend eine positive Zahl bezeichnet. In dem Aufsatze über ,die Darstellung gewisser Functionen durch die Eulersche Summenformel" im $56^{\text {sten }}$ Bande dieses Journals verificirte Herr Lipschitz die so verallgemeinerte Stirlingsche Formel und bestimmte zugleich den Rest der Reihe, die sie enthält.

Ich wurde hiedurch veranlafst eine frühere Untersuchung wieder aufzunehmen, welche zum $Z$ wecke hatte unmittelbar aus dem Integralausdruck eine convergente Reihenentwicklung für $\log T(a)$ herzustellen, und welche mich zu einem neuen Ausdruck für $\boldsymbol{\Gamma}(\boldsymbol{a})$ in Form eines unendlichen Products führte. Dieses Product ist so beschaffen, dafs, wenn $a$ wächst, die ersten Factoren unmittelbar den Näherungswerth $/ 2 \pi \cdot e^{-a} a^{a-\frac{1}{2}}$ liefern, während die übrigen Factoren gegen die Einheit convergiren, und die Entwicklung. von $\log T(a)$ aus diesem Product nimmt die Form der Stirlingschen Formel an. Man erhält hiebei zu gleicher Zeit verschiedene, so viel mir bekannt, neue independente Ausdrücke für die Bernoullischen Zahlen. Andererseits läfst sich auch aus dem Producte leicht der von Gäu/s in der oben erwähnten Abhandlung für $\Gamma(a+1)$ gegebene Ausdruck ableiten.

Durch Producte ähnlicher Art lassen sich endlich auch die Functionen $e^{\frac{\partial \log \Gamma(a)}{d a}}$ und $e^{\frac{1}{a}}$ darstellen, und zwar unterscheiden sich die Producte, welche diese beiden Functionen darstellen, nur in den Exponenten der einzelnen Factoren.

1. Nennen wir die Function $\frac{\partial \log \Gamma(a)}{\partial a}$, welche Gauss mit $\psi(a-1)$ bezeichnet, um die Formeln gleichmäfsiger zu machen, hier $\psi(a)$, so hat man nach Dirichlet (Sur les intégrales Eulériennes Bd.15, S. 260 dieses Journals) für $\psi(a)$ den Integralausdruck

(1.) $\quad \psi(a)=\int_{0}^{\infty}\left(e^{-z}-\frac{1}{(1+z)^{a}}\right) \frac{\partial z}{z}$. 
Bauer, von den Gammafunctionen u. einer besonderen Art v. Producten. 257

Aus diesem Ausdruck für $\psi(a)$ läfst sich sogleich entnehmen, dafs wenn $a$ gegen Unendlich convergirt, $\psi(a)-\log (a)$ gegen Null convergirt. In der That ist

$$
\log a=\int_{0}^{\infty}\left(e^{-z}-e^{-a z}\right) \frac{\partial z}{z}
$$

mithin

$$
\begin{aligned}
\psi(a)-\log a & =\int_{0}^{\infty}\left(e^{-a z}-\frac{1}{(1+z)^{a}}\right) \frac{\partial z}{z} \\
& =\int_{0}^{\infty}\left(e^{-y}-\frac{1}{\left(1+\frac{y}{a}\right)^{a}}\right) \frac{\partial y}{y} .
\end{aligned}
$$

Wird nun $a=\infty$, so wird $\left(1+\frac{y}{a}\right)^{-a}=e^{-y}$ in allen Elementen des Integrals, in welchen $y$ einen endlichen Werth hat; diejenigen Elemente aber, in welchen $y$ unendlich grofs ist, tragen ohnehin nichts zum Werthe des Integrals bei. Hierdurch ist obige Behauptung gerechtfertigt.

Differentiirt man die Gleichung (1.) nochmals nach $a$, und setzt

$$
\frac{\partial^{2} \log \Gamma(a)}{\partial a^{2}}=\frac{\partial \psi(a)}{\partial a}=\psi^{\prime}(a)
$$

so erhält man

$$
\begin{aligned}
\psi^{\prime}(a) & =\int_{0}^{\infty} \frac{\log (1+z)}{(1+z)^{a}} \frac{\partial z}{z}=-\int_{0}^{\infty} \frac{\log \left(1-\frac{z}{1+z}\right)}{(1+z)^{a}} \frac{\partial z}{z} \\
& =\sum_{i=1}^{i=\infty} \frac{1}{i} \int_{0}^{\infty} \frac{z^{i-1}}{(1+z)^{a+i}} \partial z .
\end{aligned}
$$

Das Integral $\int_{0}^{\infty} \frac{z^{i-1}}{(1+z)^{a+i}} \partial z$ ist aber bekanntlich $=\frac{\Gamma(i) \Gamma(a)}{\Gamma(a+i)}=\frac{1.2 .3 \ldots(i-1)}{a(a+1) \ldots(a+i-1)^{9}}$ und man hat also für $\psi^{\prime}(a)$ die Reihenentwicklung

$$
\psi^{\prime}(a)=\frac{1}{a}+\frac{1}{2} \cdot \frac{1}{a(a+1)}+\frac{1}{3} \cdot \frac{1.2}{a(a+1)(a+2)}+\frac{1}{4} \cdot \frac{1.2 .3}{a(a+1)(a+2)(a+3)}+\cdots
$$

oder

$$
\text { (2.) } \quad \psi^{\prime}(a)=\sum_{i=0}^{i=\infty} \frac{1}{i+1} \cdot \frac{1.2 .3 \ldots i}{a(a+1) \ldots(a+i)},
$$

eine Reihenentwicklung, welche convergent ist für jeden positiven Werth von $a$.

2. Gau/s hat in der schon erwähnten Abhandlung über die hypergeometrische Reihe für $\psi^{\prime}(\boldsymbol{a})$ die folgende Reihe gefunden:

$$
\psi^{\prime}(a)=\frac{1}{a^{2}}+\frac{1}{(a+1)^{2}}+\frac{1}{(a+2)^{2}}+\cdots=\sum_{i=0}^{i=\infty} \frac{1}{(a+i)^{2}} .
$$


258 Bauer, von den Gammafunctionen u. einer besonderen Art v. Producten.

Es mufs mithin für jeden positiven Werth von $a$

$$
\text { (3.) } \quad \sum_{i=0}^{i=\infty} \frac{1}{i+1} \cdot \frac{1.2 .3 \ldots i}{a(a+1) \ldots(a+i)}=\sum_{i=0}^{i=\infty} \frac{1}{(a+i)^{2}}
$$

sein. Bevor ich weiter gehe, will ich die Gleichheit dieser beiden Reihen direct beweisen.

$\mathrm{Zu}$ diesem $\mathrm{Z}$ wecke betrachten wir die allgemeinere Reihe

$$
\text { (4.) } \quad \sum_{i=1}^{i=\infty} \frac{1}{i+k} \cdot \frac{1.2 .3 \ldots i}{(a+1)(a+2) \ldots(a+i)}=f(k, a) \text {. }
$$

Schliefsen wir den Fall aus, wo $k$ eine ganze negative Zahl ist, so ist diese Reihe convergent für jeden Werth von $k$ und jeden positiven Werth von $a$. Für $k=0$ geht sie in die Reihe

$$
\frac{1}{a+1}+\frac{1}{(a+1)(a+2)}+\frac{1.2}{(a+1)(a+2)(a+3)}+\cdots
$$

über, deren Summe $=\frac{1}{a}$ ist, so dafs also

$$
\text { (5.) } f(0, a)=\frac{1}{a} \text {. }
$$

Nun folgt alls (4.)

mithin

$$
\begin{aligned}
\frac{k}{a+1} f(k, a+1) & =\sum \frac{k}{i+k} \cdot \frac{1.2 .3 \ldots i}{(a+1)(a+2) \ldots(a+i+1)} \\
\left(1-\frac{k}{a+1}\right) f(k, a+1) & =\sum \frac{a+1-k}{i+k} \cdot \frac{1.2 .3 \ldots i}{(a+1)(a+2) \ldots(a+i+1)}
\end{aligned}
$$

$$
\begin{aligned}
\left(1-\frac{k}{a+1}\right) f(k, a+1)-f(k, a) & =-\Sigma \frac{1.2 .3 \ldots i}{(a+1)(a+2) \ldots(a+i+1)} \\
& =-\left(f(0, a)-\frac{1}{a+1}\right)=\frac{-1}{a(a+1)} .
\end{aligned}
$$

Man hat also die Relation

$$
\text { (6.) } f(k, a)=\frac{1}{a(a+1)}+\frac{a-k+1}{a+1} f(k, a+1) \text {, }
$$

wodurch $f(k, a+1)$ auf $f(k, a)$ zurückgeführt wird, ausgenommen wenn $k=a+1$ ist. Andererseits giebt diese Relation durch wiederholte Substitution, da $f(k, a)$ mit wachsendem $a$ gegen Null convergirt, für $f(k, a)$ die Reihe

$$
\begin{gathered}
f(k, a)= \\
\frac{1}{a(a+1)}+\frac{a-k+1}{(a+1)^{2}(a+2)}+\frac{(a-k+1)(a-k+2)}{(a+1)(a+2)^{2}(a+3)}+\frac{(a-k+1)(a-k+2)(a-k+3)}{(a+1)(a+2)(a+3)^{2}(a+4)}+\cdots,
\end{gathered}
$$

welche abbricht, so oft $k$ um eine ganze Zahl gröfser ist als $a$. 
Für $k=1$ giebt diese Reihe

$$
f(1, a)=\frac{1}{a(a+1)}+\frac{a}{(a+1)^{2}(a+2)}+\frac{a}{(a+2)^{2}(a+3)}+\frac{a}{(a+3)^{2}(a+4)}+\cdots
$$

und für $k=0$

$$
f(0, a)=\frac{1}{a}=\frac{1}{a(a+1)}+\frac{1}{(a+1)(a+2)}+\frac{1}{(a+2)(a+3)}+\cdots,
$$

eine Gleichung, deren Richtigkeit von selbst erhellt. Aus diesen beiden Reihen folgt nun

$$
\frac{1}{a} f(1, a)+\frac{1}{a}=\frac{a+1}{a^{2}(a+1)}+\frac{a+2}{(a+1)^{2}(a+2)}+\frac{a+3}{(a+2)^{2}(a+3)}+\cdots,
$$

oder

$$
\sum_{i=0}^{i=\infty} \frac{1}{i+1} \cdot \frac{1 \cdot 2.3 \ldots i}{a(a+1) \ldots(a+i)}=\sum_{i=0}^{i=\infty} \frac{1}{(a+i)^{2}}
$$

was zu beweisen war.

3. Verweilen wir noch etwas bei der Reihe $f(k, a)$. Dieselbe läfst sich auf folgende Weise zerlegen:

$$
\begin{aligned}
f(k, a) & =\frac{1}{(1+k)(a+1)}+\sum_{i=2} \frac{1.2 \ldots i-1}{(a+1) \ldots(a+1+i-1)}-k \sum_{i=2} \frac{1}{i+k} \cdot \frac{1.2 \ldots i-1}{(a+1) \ldots(a+1+i-1)} \\
& =\frac{1}{(1+k)(a+1)}+\sum_{i=1} \frac{1.2 \ldots i}{(a+1) \ldots(a+1+i)}-k \sum_{i=1} \frac{1}{i+k+1} \cdot \frac{1.2 \ldots i}{(a+1) \ldots(a+1+i)} \\
& =\frac{1}{(1+k)(a+1)}+\frac{1}{a}-\frac{1}{a+1}-\frac{k}{a+1} f(k+1, a+1) .
\end{aligned}
$$

Es. ist also

$$
\text { (7.) } f(k, a)=\frac{1}{a}-\frac{k}{k+1} \cdot \frac{1}{a+1}-\frac{k}{a+1} f(k+1, a+1) \text {, }
$$

und aus dieser Relation und der Relation (6.) ergiebt sich

$$
\text { (8.) } \quad(a-k) f(k, a)=\frac{1}{1+k}-k f(k+1, a) \text {. }
$$

Mittelst dieser Gleichung ist $\boldsymbol{f}(\boldsymbol{k}+1, \boldsymbol{a})$ auf $\boldsymbol{f}(\boldsymbol{k}, \boldsymbol{a})$ zurückgeführt, oder umgekehrt, ausgenommen wenn $k=0$ oder $k=a$ ist. Ist also $k$ eine ganze positive Zahl, so läfst sich $f(k, a)$ auf $f(1, a)$ zurückführen und hängt mithin von $\psi^{\prime}(a)$ ab. Eine ähnliche Aenderung wie die, welche die Function $f(k, a)$ erleidet beim Uebergang von $k=0$ auf $k=1$, erleidet dieselbe auch beim Uebergang von $k=a+1$ auf $k=a$. Für $k=a+1$, wie überhaupt für $\boldsymbol{k}=\boldsymbol{a}+\boldsymbol{n}$, wo $\boldsymbol{n}$ eine ganze positive Zahl, ist $\boldsymbol{f}(\boldsymbol{k}, \boldsymbol{a})$ eine rationale Function von $a$ (nach $\mathrm{Nr} .2$ ), für $k=a$ hingegen hängt der Werth von $f(k, a)$ ebenfalls von $\psi^{\prime}(a)$ ab, wie aus einer bemerkenswerthen Transformation mittelst 
260 Bauer, von den Gammafunctionen u. einer besonderen Art v. Producten.

der Relation (7.) hervorgeht. Da nämlich, wenn $k$ und $a$ wächst, $f(k, a)$ gegen Null convergirt, so folgt aus dieser Relation

$$
\begin{aligned}
f(k, a)=\frac{1}{a}-\frac{k}{k+1} \cdot \frac{1}{a+1}-\frac{k}{a+1}\left(\frac{1}{a+1}-\frac{k+1}{k+2} \cdot \frac{1}{a+2}-\frac{k+1}{a+2}\left(\frac{1}{a+2}-\cdots\right.\right. \\
=\frac{1}{a}-k\left(\frac{1}{(k+1)(a+1)}+\frac{1}{(a+1)^{2}}\right)+\frac{k(k+1)}{a+1}\left(\frac{1}{(k+2)(a+2)}+\frac{1}{(a+2)^{2}}\right) \\
\quad-\frac{k(k+1)(k+2)}{(a+1)(a+2)}\left(\frac{1}{(k+3)(a+3)}+\frac{1}{(a+3)^{2}}\right)+-\cdots
\end{aligned}
$$

Mithin für $k=a$

oder auch

$$
\begin{aligned}
f(a, a) & =\frac{1}{a}-\frac{2 a}{(a+1)^{2}}+\frac{2 a}{(a+2)^{2}}-\frac{2 a}{(a+3)^{2}}+-\cdots \\
& =-\frac{1}{a}+2 a \sum_{i=0}^{i=\infty} \frac{(-1)^{i}}{(a+i)^{2}}
\end{aligned}
$$

(9.) $\quad \sum_{i=0}^{i=\infty} \frac{1}{a+i} \cdot \frac{1 \cdot 2.3 \ldots i}{a(a+1) \ldots(a+i)}=2 \sum_{i=0}^{i=\infty} \frac{(-1)^{i}}{(a+i)^{2}}$.

Da nun

$$
\Sigma \frac{(-1)^{i}}{(2 a+i)}=\frac{1}{2} \Sigma \frac{1}{(a+i)^{2}}-\Sigma \frac{1}{(2 a+1)^{2}}
$$

ist und $\Sigma \frac{1}{(a+i)^{2}}=\psi^{\prime}(a)$, so wird

$$
\sum_{i=1}^{i=\infty} \frac{1}{a+i} \cdot \frac{1.2 \ldots i}{a(a+1) \ldots(a+i)}=\psi^{\prime}\left(\frac{a}{2}\right)-2 \psi^{\prime}(a) .
$$

Aus dem Vorhergehenden ergiebt sich also, dafs die Transcendente $f(k, a)$ als bekannt angesehen werden kann für jeden Werth von $k$ und jeden positiven Werth von $a$, wenn man sie für jeden Werth von $k$ und $a$ zwischen 0 und 1 kennt; ferner dafs sie eine rationale Function von $a$ wird, wenn $\boldsymbol{k}=\mathbf{0}$ oder wenn $\boldsymbol{k}$ um eine ganze Zahl gröfser als $a$ ist; hingegen auf die Function $\frac{\partial^{2} \log \Gamma(a)}{\partial a^{2}}=\psi^{\prime}(a)$ zurückgeführt werden kann, wenn $k$ eine ganze positive Zahl ist, so wie auch, wenn $k=a$ oder um eine ganze Zahl kleiner als $a$ ist.

4. Kehren wir nun zur Gleichung (2.) zurück. Die Reihe, durch welche dort $\psi^{\prime}(\boldsymbol{a})$ dargestellt ist, ist zwar weniger einfach, als die von Gau/s gegebene, allein sie hat vor dieser den Vorzug, dafs sie bei zweimaliger Integration zu convergenten Reihen führt.

Zerlegen wir den Ausdruck $\frac{1.2 .3 \ldots i}{a(a+1) \ldots(a+i)}$ in Partialbrüche nach bekannten Formeln, so erhalten wir 
Bauer, von den Gammafunctionen u. eirer besonderen Art v. Producten. 261 (10.) $\frac{1.2 .3 \ldots i}{a(a+1) \ldots(a+i)}=\frac{1}{a}-\left(\begin{array}{c}i \\ 1\end{array}\right) \frac{1}{a+i}+\left(\begin{array}{c}i \\ 2\end{array}\right) \frac{1}{a+2}-\left(\begin{array}{l}i \\ 3\end{array}\right) \frac{1}{a+3}+-\cdots \pm \frac{1}{a+i}$, wo $\left(\begin{array}{l}i \\ 1\end{array}\right),\left(\begin{array}{l}i \\ 2\end{array}\right)$ u. s. f. den ersten, zweiten u. s. f. Binomialcoefficienten der $i^{\text {ten }}$ Potenz bezeichnet.

Die Integration der G!eichung (2.) giebt mithin

$$
\begin{gathered}
\psi(a)= \\
\sum_{i=0}^{i=\infty} \frac{1}{i+1}\left[\log \alpha-\left(\begin{array}{c}
i \\
1
\end{array}\right) \log (a+1)+\left(\begin{array}{c}
i \\
2
\end{array}\right) \log (a+2)-+\cdots \pm \log (a+i)\right]+\text { const. }
\end{gathered}
$$

Wir werden aber in $\mathrm{Nr} .5$ sehen, dafs die Constante der Integration hier Null ist, und es wird mithin

$$
\text { (11.) } \psi(\boldsymbol{a})=\log \prod_{i=0}^{i=\infty}\left[\frac{a(a+2))^{\left(\begin{array}{c}
i \\
2
\end{array}\right)} \ldots}{(a+1)^{\left(\begin{array}{l}
i \\
1
\end{array}\right)}(a+3)^{\left(\begin{array}{c}
i \\
3
\end{array}\right)} \ldots}\right]^{\frac{1}{i+1}},
$$

wo $I I$ das unendliche Product anzeigt. Die einzelnen Glieder dieses Producls hängen auf sehr einfache Weise von einander ab. Nennen wir die Gröfse in der Klammer $\varphi_{i}$, so dafs

so ist

$$
\text { (11'.) } \quad \psi(\boldsymbol{a})=\log \left[q_{0} \cdot q_{1}^{\frac{1}{2}} \cdot \varphi_{2}^{\frac{1}{3}} \cdot q_{3}^{\frac{1}{4}} \ldots\right],
$$

$$
q_{0}=a, \quad q_{1}=\frac{a}{a+1}
$$

und allgemein ist, wenn wir mit $\boldsymbol{q}_{i}^{\prime}$ das bezeichnen, was $\boldsymbol{q}_{i}$ wird, wenn man darin $a+1$ statt $a$ setzt

$$
\boldsymbol{q}_{i+1}=\frac{\boldsymbol{q}_{i}}{\boldsymbol{q}_{i}^{\prime}},
$$

wie sich aus der bekannten Eigenschaft der Binomialcoefficienten, nach wel$\operatorname{cher}\left(\begin{array}{c}i \\ n\end{array}\right)+\left(\begin{array}{c}i \\ n+1\end{array}\right)=\left(\begin{array}{c}i+1 \\ n+1\end{array}\right)$ ist, sogleich ergiebt.

5. Untersuchen wir umgekehrt die Convergenz des Ausdrucks für $\psi(a)$. Vermöge der Relation $\left(\begin{array}{c}i \\ n\end{array}\right)=\left(\begin{array}{c}i-1 \\ n\end{array}\right)+\left(\begin{array}{c}i-1 \\ n-1\end{array}\right)$ hat man von $i=1$ an

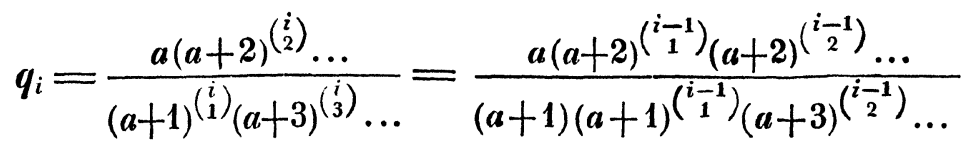

$$
\begin{aligned}
& =\frac{\left(1+\frac{1}{a+1}\right)^{\left(\frac{i-1}{1}\right)}\left(1+\frac{1}{a+3}\right)^{\left(\frac{i-1}{3}\right)} \cdots}{\left(1+\frac{1}{a}\right)\left(1+\frac{1}{a+2}\right)^{\left(\begin{array}{c}
i-1 \\
2
\end{array}\right)} \cdots} .
\end{aligned}
$$

Journal für Mathematik Bd. LVII. Heft 3. 
Bezeichnen wir nun zur Abkürzung die Gröfsen $\frac{1}{a}, \frac{1}{a+1}, \frac{1}{a+2}, \ldots$ der Reihe nach mit $\alpha_{0}, \alpha_{1}, \alpha_{2}, \ldots$ und bilden wir das System von Gleichungen

$$
\begin{aligned}
& \frac{1+\alpha_{0}}{1+\alpha_{1}}=1+\beta_{0}, \quad \frac{1+\alpha_{1}}{1+\alpha_{2}}=1+\beta_{1}, \quad \frac{1+\alpha_{2}}{1+\alpha_{3}}=1+\beta_{2}, \ldots \\
& \frac{1+\beta_{0}}{1+\beta_{1}}=1+\gamma_{0}, \quad \frac{1+\beta_{1}}{1+\beta_{2}}=1+\gamma_{1}, \ldots \ldots \ldots \\
& \frac{1+\gamma_{0}}{1+\gamma_{1}}=1+\delta_{0}
\end{aligned}
$$

so sind $\beta_{0}, \beta_{1}, \beta_{2}, \ldots ; \gamma_{0}, \gamma_{1}, \gamma_{2}, \ldots ; \delta_{0}, \delta_{1}, \delta_{2}, \ldots$ u. s. f. Reihen, deren Glieder respective aus $\beta_{0}, \gamma_{0}, \delta_{0}$ u. s. w. hervorgehen, wenn man darin $a$ nach und nach durch $a+1, a+2$, u. s. w. ersetzt, und sämmtlich von der positiven Seite her gegen die Null convergiren.

Nun folgt aus obigen Gleichungen

und hieraus

$$
\beta_{0}=\frac{\alpha_{0}-\alpha_{1}}{1+\alpha_{1}}, \quad \gamma_{0}=\frac{\beta_{0}-\beta_{1}}{1+\beta_{1}}, \quad \delta_{0}=\frac{\gamma_{0}-\gamma_{1}}{1+\gamma_{1}}, \quad \text { u. s. f. }
$$

$$
\begin{aligned}
& \beta_{0}=\left(\alpha_{0}-\alpha_{1}\right)-\alpha_{1} \beta_{0}, \\
& \gamma_{0}=\left(\beta_{0}-\beta_{1}\right)-\beta_{1} \gamma_{0}=\left(\alpha_{0}-2 \alpha_{1}+\alpha_{2}\right)-\left(\alpha_{1} \beta_{0}-\alpha_{2} \beta_{1}\right)-\beta_{1} \gamma_{0}, \\
& \delta_{0}=\left(\gamma_{0}-\gamma_{1}\right)-\gamma_{1} \delta_{0}=\left(\alpha_{0}-3 \alpha_{1}+3 \alpha_{2}-\alpha_{3}\right)-\left[\left(\alpha_{1} \beta_{0}-\alpha_{2} \beta_{1}\right)-\left(\alpha_{2} \beta_{1}-\alpha_{3} \beta_{2}\right)\right] \\
& \text { u. s. f. } \quad-\left(\beta_{1} \gamma_{0}-\beta_{2} \gamma_{1}\right)-\gamma_{1} \delta_{0},
\end{aligned}
$$

Die ersten Glieder dieser Ausdrücke für $\beta_{0}, \gamma_{0}, \delta_{0}, \ldots$ sind nach Gleichung (10.)

$$
\frac{1}{a(a+1)}, \frac{1.2}{a(a+1)(a+2)}, \frac{1.2 .3}{a(a+1)(a+2)(a+3)}, \ldots
$$

Hieran reihen sich, wie man sogleich sieht, die folgenden Glieder in absteigender Ordnung. So sind die zweiten Glieder $\alpha_{1} \beta_{0}=\frac{1}{a(a+1)(a+2)}$ und seine Differenzen, die dritten Glieder $\beta_{1} \gamma_{0}=\frac{1}{(a+2)^{2}}\left(\frac{1}{a(a+2)}-\frac{1}{(a+1)(a+3)}\right)$ und seine Differenzen u. s. f. Es ergiebt sich mithin aus diesen Gleichungen, dafs die Gröfsen $\beta_{0}, \gamma_{0}, \delta_{0}, \ldots$ positiv sind und zwar

$$
\beta_{0}<\frac{1}{a(a+1)}, \quad \gamma_{0}<\frac{1.2}{a(a+1)(a+2)}, \quad \delta_{0}<\frac{1.2 .3}{a(a+1)(a+2)(a+3)}, \quad \text { u. s. f. }
$$

Nun ist aber

$$
1+\beta_{0}=\frac{1}{q_{2}}, \quad 1+\gamma_{0}=\frac{1}{q_{3}}, \quad 1+\delta_{0}=\frac{1}{q_{4}}, \text { u. s. f. }
$$


Bauer, von den Gammafunctionen u. einer besonderen Art v. Producten. 263 und also allgemein

$$
\frac{1}{\eta_{i}}=1+\varepsilon \frac{1.2 \ldots(i-1)}{a(a+1) \ldots(a+i-1)},
$$

wo $\varepsilon$ ein ächter positiver Bruch ist. Es ist folglich auch $\log q_{i}^{\frac{1}{i+1}}$ numerisch

$$
<\frac{1}{i+1} \cdot \frac{1.2 \ldots(i-1)}{a(a+1) \ldots(a+i-1)},
$$

woraus die Convergenz des Products $\Pi \boldsymbol{q}_{i}^{\frac{1}{i+1}}$ für jeden positiven Werth von $a$ erhellt. Zugleich ersieht man, dafs $q_{i}<1$ ist für jedes $i$ von $i=1$ an.

Es läfst sich nun auch leicht zeigen, dafs die Constante der Integration in der Gleichung (11.) für $\psi(a)$ Null ist, wie ich dort erwähnte. Es ist nämlich nach dem Vorhergehenden $\sum_{i=1}^{i=\infty} \log \eta_{i}^{\frac{1}{i+1}}$ numerisch $<\frac{1}{2} \sum \frac{1.2 \ldots i-1}{a(a+1) \ldots(a+i-1)}$ oder nach $(5)<.\frac{1}{2} \cdot \frac{1}{a-1}$. Für $a=\infty$ convergirt also diese. Summe gegen Null. Dasselbe ist aber auch der Fall mit $\psi(a)-\log a$, wie wir in Nr. 1 sahen. Folglich mufs die Constante der Integration in (11.) Null sein.

6. Integrirt man nun wiederum die Gleichung (11.), so erhält man auf der rechten Gleichungsseite nur Glieder der Form $\left(\begin{array}{l}i \\ n\end{array}\right)[(a+n) \log (a+n)-a]$. Aber vermöge der Relation $1-\left(\begin{array}{c}i \\ 1\end{array}\right)+\left(\begin{array}{c}i \\ 2\end{array}\right)-\left(\begin{array}{c}i \\ 3\end{array}\right)+-\cdots=0$, welche für jede positive ganze Zahl $i$ gilt, heben sich die Vielfachen von $a$ auf für jedes $i$, aufser für $i=0$, und man erhält

$$
\log \Gamma(a)=\log C+a \log a-a
$$

$+\sum_{i=1}^{i=\infty} \frac{1}{i+1}\left[a \log a-\left(\begin{array}{l}i \\ 1\end{array}\right)(a+1) \log (a+1)+\left(\begin{array}{l}i \\ 2\end{array}\right)(a+2) \log (a+2)-\cdots(a+i) \log (a+i)\right]$ oder

$$
\text { (12.) } \quad \boldsymbol{I}(\boldsymbol{a})=\boldsymbol{C} \cdot \boldsymbol{e}^{-a} \prod_{i=0}^{i=0}\left[\frac{a^{a}(a+2)^{\left(\frac{i}{2}\right)(a+2)} \ldots}{(a+1)^{(i)(a+1)}(a+3)^{\left(\frac{i}{3}\right)(a+3)} \ldots}\right]^{\frac{1}{i+1}},
$$

wo $C$ die Constante der Integration ist. Bezeichnen wir die Glieder dieses Products, abgesehen vom Exponenten $\frac{1}{i+1}$, mit $\boldsymbol{Q}_{0}, \boldsymbol{Q}_{1}$, u. s. f., so ist

und man hat

$$
\left(12^{\prime} .\right) \quad \Gamma(a)=C e^{-a} Q_{0} Q_{1}^{\frac{1}{2}} Q_{2}^{\frac{1}{3}} Q_{3}^{\frac{1}{4}} \ldots
$$

und allgemein

$$
\boldsymbol{Q}_{0}=\boldsymbol{a}^{a}, \quad \boldsymbol{Q}_{1}=\frac{\boldsymbol{a}^{a}}{(a+1)^{(a+1)}}
$$


264 Bauer, von den Gammafunctionen u. einer besonderen Art v. Producten.

$$
\boldsymbol{Q}_{i+1}=\frac{\boldsymbol{Q}_{i}}{\boldsymbol{Q}_{i}^{\prime}}
$$

wenn wieder $\boldsymbol{Q}_{i}^{\prime}$ das bezeichnet, was $\boldsymbol{Q}_{i}$ wird, wenn man darin $\boldsymbol{a}+1$ statt a setzt.

Obiger Ausdruck für $I^{\prime}(a)$ ist ebenfalls convergent für jeden positiven Werth von $\boldsymbol{a}$. Denn fafst man in $\boldsymbol{Q}_{i}$ alle Factoren zusammen, welche $\boldsymbol{a}$ im Exponenten haben, so erhält man

$$
\boldsymbol{Q}_{i}=\left[\frac{\left.a(a+2)^{(i)}\right) \ldots}{(a+1)^{\left(\frac{i}{1}\right)}(a+3)^{\left(\frac{i}{3}\right)} \ldots}\right]^{a} \cdot\left[\frac{(a+2)^{\left(\frac{i-1}{1}\right)} \ldots}{(a+1)(a+3)^{\left(\frac{i-1}{2}\right)} \ldots}\right]^{i},
$$

da allgemein $n\left(\begin{array}{l}i \\ n\end{array}\right)=i\left(\begin{array}{l}i-1 \\ n-1\end{array}\right)$ ist. Nennen wir den ersten Factor $\boldsymbol{A}_{i}$, den zweiten $B_{i}$, so ist nach Nr. $5 \log A_{i}^{\frac{1}{i+1}}$ negativ und numerisch

$$
<\frac{1}{i+1} \cdot \frac{1.2 \ldots(i-1)}{(a+1)(a+2) \ldots(a+i-1)},
$$

hingegen, von $i=2$ an, $\log B_{i}^{\frac{1}{i+1}}$ positiv und

Die beiden Producte

$$
<\frac{i}{i+1} \cdot \frac{1.2 \ldots(i-2)}{(a+1)(a+2) \ldots(a+i-1)} .
$$

\section{$\Pi \boldsymbol{A}_{i}^{\frac{1}{i+1}}$ und $\Pi \boldsymbol{B}_{i}^{\frac{1}{i+1}}$}

sind mithin convergent und folglich auch das Product $\Pi \boldsymbol{Q}_{i}^{\frac{1}{i+1}}$. Man wird zugleich bemerken, dafs $Q_{i}$ von $i=2$ an immer $>1$ ist, während $Q_{1}$ natürlich immer $<1$ ist.

Um nun die Constante der Integration $C$ in Gleichung (12.) zu bestimmen, lassen wir $a$ gegen $\infty$ convergiren, so convergirt $\log \prod_{i=2}^{i=\infty} \boldsymbol{Q}_{i}^{\frac{1}{i+1}}$ gegen Null, also $\prod_{i=2}^{i=\infty} Q_{i}^{\frac{1}{i+1}}$ gegen die Einheit; $Q_{1}=\frac{1}{\left(1+\frac{1}{a}\right)^{a}} \cdot \frac{1}{a+1}$ convergirt gegen $\frac{1}{e a}$ und es wird sodann

$$
\text { (13.) } \quad \Gamma(a)=C e^{-a} a^{a}\left(\frac{1}{e a}\right)^{\frac{1}{2}}=C \frac{a^{a-\frac{1}{2}}}{e^{a+\frac{1}{2}}}
$$

oder auch, wenn man $a+1$ an die Stelle von $a$ setzt,

$$
\text { (13'.) } \quad \Gamma(a+1)=C\left(\frac{a}{e}\right)^{a+\frac{1}{2}} .
$$


Bauer, von den Gammafunctionen u. einer besonderen Art v. Producten. 265

Mittelst dieser Gleichung und der Formel von Wallis ist es nun leicht die Constante $\boldsymbol{C}$ zu bestimmen. Denn bekanntlich ergiebt sich unmiltelbar' aus dem Integral, das $\Gamma(a)$ darstellt, die Gleichung $\boldsymbol{I}(\boldsymbol{a}+1)=a \Gamma(a)$, und wir werden später (Nr. 8) sehen, dafs diese Eigenschaft der Function $T$ anch leicht aus Gleichung (12.) geschlossen werden kann. Aus dieser Eigenschaft folgt, wenn $x$ eine ganze Zahl ist, $\Gamma(x+1)=1.2 .3 \ldots x$ und mithin, wenn $x$ gegen Unendlich convergirt, 1.2.3...x=C( $\left.\frac{x}{e}\right)^{x+\frac{1}{2}}$. Die Formel von Wallis

$$
\frac{\pi}{2}=\lim \cdot \frac{2.2 .4 .4 \ldots(2 x-2) 2 x}{1.3 .3 .5 \ldots(2 x-1)(2 x-1)}
$$

giebt folglich, wenn man bemerkt, dafs

$$
1.2 .3 \ldots 2 x=2^{x} \cdot 1.2 .3 \ldots x .1 .3 .5 \ldots(2 x-1)
$$

ist,

woraus

$$
\frac{\pi}{2}=\lim \cdot \frac{1}{2 x} \frac{\left(1.2 .3 \ldots x \cdot 2^{x}\right)^{4}}{(1.2 .3 \ldots 2 x)^{2}}=\frac{C^{2}}{4 e}
$$

sich ergiebt.

$$
\text { (14.) } C=\sqrt{2 \pi e}
$$

7. Wie aus der Reihe $\sum \frac{1}{i+k} \frac{1.2 \ldots i}{a(a+1) \ldots(a+i)}$ für $k=1$ sich die unendlichen Producte für $e^{\psi(a)}$ und $\Gamma(a)$ ergaben, ebenso erhält man für $k=0$ den Werth anderer ähnlicher Producte. Es wird nämlich für $k=0$

$$
\text { (15.) } \quad \sum_{i=1}^{i=\infty} \frac{1}{i} \frac{1.2 .3 \ldots i}{a(a+1) \ldots(a+i)}=\frac{1}{a^{2}}
$$

da diese Reihe mit der Reihe (5.) zusammenfällt, wenn man lelztere mit $\frac{1}{a}$ multiplicirt. Die Integration dieser Gleichung giebt

$$
-\frac{1}{a}=\sum_{i=1}^{i=\infty} \frac{1}{i}\left[\log a-\left(\begin{array}{c}
i \\
1
\end{array}\right) \log (a+1)+\left(\begin{array}{c}
i \\
2
\end{array}\right) \log (a+2)-+\cdots\right]
$$

oder

$$
\text { (16.) } e^{-\frac{1}{a}}=\prod_{i=1}^{i=\infty}\left[\frac{a(a+2)^{\left(\begin{array}{l}
i \\
2
\end{array}\right)} \ldots}{(a+1)^{\left(\begin{array}{l}
i \\
1
\end{array}\right)}(a+3)^{\left(\begin{array}{c}
i \\
3
\end{array}\right)} \ldots}\right]^{\frac{1}{i}} \text {. }
$$

Dieses Product ist nach Nr. 5 ebenfalls convergent für jedes positive a. Die Constante der Integration ist Null, indem für $a=\infty$ beide Seiten der Gleichung (16.) gegen die Einheit convergiren. Führt man die Gröfsen $q_{i}$ ein, so ist

$$
\left(16^{\prime} .\right) \quad e^{-\frac{1}{a}}=q_{1} \cdot q_{2}^{\frac{1}{2}} \cdot q_{3}^{\frac{1}{3}} \cdot q_{4}^{\frac{1}{4}} \cdots
$$


266 Bauer, von den Gammafunctionen u. ciner besonderen Art v. Producten.

und dieses Product unterscheidet sich nur durch die Exponenten $\frac{1}{i}$ von dem Product in $\left(11^{\prime}.\right)$, welches $e^{\psi(a)}$ darstellt.

Geht man unmittelbar von der Reihe (5.) aus, so erbält man auf gleiche Weise

oder auch

$$
\text { (17.) } \quad \boldsymbol{a}=\prod_{i=0}^{i=\infty}\left[\frac{(a+1)(a+3)^{(i)} \ldots}{(a+2)^{(i)}(a+4)^{\left(\frac{i}{(i)}\right)} \ldots}\right]
$$

$$
a=q_{0}^{\prime} \cdot q_{1}^{\prime} \cdot q_{2}^{\prime} \cdot q_{3}^{\prime} \ldots
$$

wo $q_{i}^{\prime}$ wie früher das bezeichnet, was $q_{i}$ wird, wenn man darin $a+1$ statt $a$ setzt, und auch dieses Product ist. nach Nr. 5 convergent für jedes positive $a$, indem $\log q_{i}^{\prime}$ numerisch $<\frac{1.2 \ldots i-1}{(a+1)(a+2) \ldots(a+i)}$ ist.

Von der Reihe (5.) läfst sich der Rest angeben. Bricht man dieselbe vor dem $n^{\text {teu }}$ Gliede ab, so ist derselbe $\frac{1.2 .3 \ldots n}{a(a+1)(a+2) \ldots(a+n)}$. Hieraus ergiebt sich die allgemeinere Gleichung

$$
\text { (18.) } \frac{a(a+2)^{\left(\begin{array}{l}
n \\
(2)
\end{array}\right.} \ldots}{(a+1)^{\left(\begin{array}{l}
n \\
1
\end{array}\right)}(a+3)^{\left(\frac{n}{3}\right)} \ldots}=\prod_{i=n}^{i=\infty} \frac{(a+1)(a+3)^{\left(\begin{array}{l}
(i) \\
2
\end{array}\right) \ldots}}{(a+2)^{\left(\begin{array}{l}
i \\
1
\end{array}\right)}(a+4)^{\left(\frac{i}{3}\right)} \ldots},
$$

welche die Gleichung (17.) als speciellen Fall in sich schliefst.

Mit Hülfe der Gleichung (18.) in Verbindung mit dem, was in Nr. 5 gesagt ist, lassen sich nun leicht Grenzen für den Fehler angeben, den man begeht, wenn man die bisher gegebenen Producte bei irgend einem Gliede abbricht. Ich will aber hierbei nicht verweilen und nur noch bemerken, dafs man die Zahl $\boldsymbol{e}$ auch durch die Gröfsen $\boldsymbol{Q}$ darstellen kann. Die Gleichung (17.) nämlich läfst sich auch so schreiben

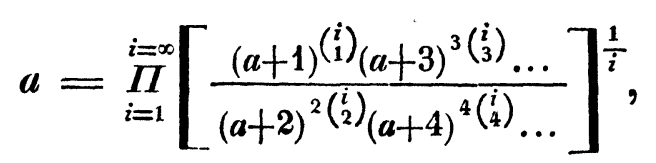

und wenn man mit dieser Gleichung die Gleichung (16.) dividirt, nachdem man letzlere in die $a^{\text {te }}$ Potenz erboben hat, so kommt

oder

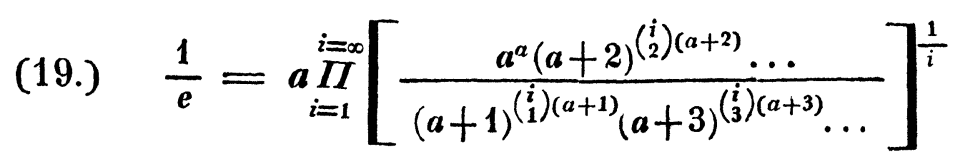

$$
\frac{1}{e}=a Q_{1} Q_{2}^{\frac{1}{2}} Q_{3}^{\frac{1}{3}} Q_{4}^{4} \ldots
$$


Bauer, von den Gammafunctionen u. einer besonderen Art v. Producten. 267

Wie die beiden ersten Glieder des Products in (12.) für grofse Werthe von $a$ den Näherungswerth $C\left(\frac{a}{e}\right)^{a+\frac{1}{2}}$ für $\Gamma(a+1)$ liefern, indem das Product der folgenden Factoren gegen die Einheit convergirt, ebenso geben die ersten Factoren der Producte in (16.) und (19.) den bekannten Grenzausdruck $\left(1+\frac{1}{a}\right)^{a}$ für $e$; und zwar giebt die erstere Gleichung $e=\left(\frac{a+1}{a}\right)^{a}=\left(1+\frac{1}{a}\right)^{a}$ zu klein, die letztere $e=\left(\frac{a+1}{a}\right)^{a+1}=\left(1+\frac{1}{a}\right)^{a+1}$ zu grofs, wie man aus der Natur der folgenden Factoren ersieht.

8. Mittelst der Formeln der vorhergehenden $\mathrm{Nr}$. ist es nun leicht die Producte in (11.) und (12.) umzuformen und daraus die Gau/sschen Formeln abzuleiten.

Es war

$$
\Gamma(a)=C e^{-a} \prod_{i=0}^{i=\infty} Q_{i}^{\frac{1}{i+1}}
$$

und Gleichung (19.) läfst sich schreiben

$$
\frac{1}{c}=a \prod_{i=0}^{i=\infty} Q_{i+1}^{\frac{1}{i+1}}
$$

Die Division der ersten Gleichung durch die zweite giebt unmittelbar

$$
\Gamma(a)=\frac{C}{a} e^{-(a+1)} \prod_{i=0}^{i=\infty} Q_{i}^{\frac{1}{i+1}}
$$

wenn man wie früher mit $Q_{i}^{\prime}$ das bezeichnet, was $Q_{i}$ wird, wenn man $a+1$ statt $a$ selzt und bemerkt, dafs $\boldsymbol{Q}_{i+1}=\frac{Q_{i}}{Q_{i}^{\prime}}$ ist. Setzt man für $C e^{-(a+1)} \Pi Q_{i}^{\frac{1}{i+1}}$ seinen Werth $\Gamma(a+1)$, so giebt die letzte Gleichung die Fundamentalrelation $\Gamma(a+1)=a \Gamma(a)$.

Setzt man nun

$$
\frac{1}{e}=(a+1) \prod_{i=0}^{i=\infty} Q_{i+1}^{\frac{1}{i+1}}
$$

und dividirt die letzte Gleichung mit dieser, so erhält man

$$
\Gamma(a)=\frac{C}{a(a+1)} e^{-(a+2)} \Pi Q_{i}^{\prime \prime} \frac{1}{i+1},
$$

wo $Q_{i}^{\prime \prime}$ das bedeutet, was $\boldsymbol{Q}_{i}^{\prime}$ wird, wenn man $a+1$ statt a setzt, oder das, was $\boldsymbol{Q}_{i}$ wird, wenn $a+2$ statt $a$ gesetzt wird. So fortschreitend erhält man nach $n$ Transformationen

$$
\Gamma(a)=\frac{C}{a(a+1) \ldots(a+n-1)} e^{-(a+n)} \Pi Q_{i}^{(n) \frac{1}{i+1}}=\frac{\Gamma(a+n)}{a(a+1) \ldots(a+n-1)} .
$$


268 Bauer, von den Gammafunctionen u. einer besonderen Art v. Producten.

Läfst man $n$ gegen Unendlich convergiren und setzt für $\boldsymbol{T}(\boldsymbol{a}+\boldsymbol{n})$ seinen Grenzwerth nach (13.) $=C \frac{(a+n)^{a+n-\frac{1}{2}}}{e^{a+n+\frac{1}{2}}}=C \frac{n^{a+n-\frac{1}{2}}}{e^{n+\frac{1}{3}}}$, so wird

$$
\Gamma(a)=\frac{n^{a-1}}{a(a+1) \ldots(a+n-1)} \boldsymbol{C}\left(\frac{n}{e}\right)^{n+\frac{1}{2}}
$$

und diese Gleichung dividirt mit dem entsprechenden Ausdruck für $I^{\prime}(1)$ giebt die Formel von Gau/s

$$
\Gamma(a)=\lim \cdot \frac{1.2 .3 \ldots n \cdot n^{a-1}}{a(a+1)(a+2) \ldots(a+n-1)} .
$$

Auf gleiche Weise läfst sich die Formel für $\psi(a)$ mittelst Gleichung (16.) umformen. Man hat nämlich

$$
\psi(\boldsymbol{a})=\log \prod_{i=0}^{i=\infty} q_{i}^{\frac{1}{i+1}}
$$

und nach Gleichung (16.)

$$
-\frac{1}{a}=\log \underset{i=0}{i=\infty} q_{i+1}^{i+1}
$$

Die beiden Gleichungen von einander abgezogen geben

$$
\psi(\boldsymbol{a})+\frac{1}{\boldsymbol{a}}=\log \underset{i=0}{i=\infty} \boldsymbol{\eta}_{i}^{\prime i+1}=\psi(\boldsymbol{a}+1) .
$$

wenn $q_{i}^{\prime}$ die ihm früher beigelegle Bedeutung hat und folglich $q_{i+1}=\frac{q_{i}}{q_{i}^{\prime}}$ ist.

Nach $n$ Transformationen hat man

$$
\psi(\boldsymbol{a})+\frac{1}{a}+\frac{1}{a+1}+\cdots+\frac{1}{a+n-1}=\log \prod_{i=0}^{i=\infty} q_{i}^{(n)} \frac{1}{i+1}=\psi(\boldsymbol{a}+\boldsymbol{n}) .
$$

Für $\boldsymbol{n}=\boldsymbol{\infty}$ reducirt sich das Product auf sein erstes Glied $\boldsymbol{q}_{0}^{(n)}=\boldsymbol{a}+\boldsymbol{n}$ und die vorstehende Gleichung verwandelt sich in die von Gaus gegebene Formel

$$
\psi^{\prime}(\boldsymbol{a})=\lim .\left(\log (\boldsymbol{a}+\boldsymbol{n})-\frac{1}{a}-\frac{1}{a+1}-\cdots-\frac{1}{a+n-1}\right) .
$$

9. Die hier betrachteten Producte lassen noch eine andere Transformation zu, welche die Exponenten der einzelnen Glieder ändert und die Producte etwas schneller convergirend macht. Denn da

$$
\boldsymbol{I}(\boldsymbol{a})=\boldsymbol{C} \boldsymbol{e}^{-\boldsymbol{a}} \boldsymbol{a}^{a} \prod_{i=1}^{i=\infty} \boldsymbol{Q}_{i}^{\frac{1}{i+1}} \quad \text { und } \quad \frac{1}{\boldsymbol{e}}=\boldsymbol{a} \underset{i=1}{i=\infty} \boldsymbol{Q}_{i}^{\frac{1}{i}},
$$

so erhält man durch Division beider Gleichungen

$$
\text { (20.) } \quad \Gamma(a)=C e^{-(a+1)} a^{a-1} \prod_{i=1}^{i=\infty} Q_{i}^{\frac{-1}{i(i+1)}} \text {. }
$$


Bauer, von den Gammafunctionen u. einer besonderen Art v. Producten. 269

Setzt man in dieser Gleichung $a+1$ stalt $a$, also auch $\boldsymbol{Q}_{i}^{\prime}$ statt $\boldsymbol{Q}$ und dividirt mit der so erhaltenen Gleichung die Gleichung (20.), so wird

$$
\frac{1}{e}=\left(\frac{a}{a+1}\right)^{a} \prod_{i=1}^{i=\infty} \boldsymbol{Q}_{i+1}^{\frac{-1}{i(i+1)}} \text { oder } \quad e=\left(\frac{a+1}{a}\right)^{a} \underset{i=1}{i=\infty} \boldsymbol{Q}_{i+1}^{\frac{1}{i(i+1)}},
$$

und die Verbindung dieser Gleichung mit Gleichung (20.) giebt

$$
\text { (21.) } \quad \Gamma(a)=C e^{-(a+2)} \frac{(a+1)^{\frac{3}{2} a+\frac{1}{2}}}{a^{\frac{1}{2} a+1}} \prod_{i=1}^{i=\infty} Q_{i+1}^{\frac{1.2}{i(i+1)(i+2)}} \text {. }
$$

Man sieht, wie man so weiter fortschreiten und $\Gamma(a)$ durch ein Product darstellen könnte, in welchem die Glieder Exponenten von der Form $\frac{1.2 .3 \ldots n}{i(i+1) \ldots(i+n)}$ haben.

Dieselbe Transformation läfst sich auch auf die Producte in (11.) und (16.) anwenden, welche $e^{\psi(a)}$ und $e^{-\frac{1}{a}}$ darstellen.

10. Es bleibt nun noch zu zeigen, wie aus dem für $\Gamma(a)$ gefundenen Ausdruck sich $\log I^{\prime}(a)$ in eine Reihe nach Potenzen von $\frac{1}{a}$ entwickeln läfst.

Ersetzen wir in der aus Gleichung (12.) für $\log \Gamma(a)$ sich ergebenden Reihe allgemein $\log (a+n)$ durch $\log a+\log \left(1+\frac{n}{a}\right)$, so erhält man

$$
\log \Gamma(a)
$$

$=\log C+a \log a-a+\sum_{i=1}^{i=\infty} \frac{1}{i+1}\left[a-\left(\begin{array}{c}i \\ 1\end{array}\right)(a+1)+\left(\begin{array}{c}i \\ 2\end{array}\right)(a+2)-\cdots \pm(a+i)\right] \log a$

$$
+\sum_{i=1}^{i=\infty} \frac{1}{i+1}\left[-\left(\begin{array}{c}
i \\
1
\end{array}\right)(a+1) \log \left(1+\frac{1}{a}\right)+\left(\begin{array}{l}
i \\
2
\end{array}\right)(a+2) \log \left(1+\frac{2}{a}\right)-+\cdots\right] .
$$

Nun ist aber für jedes $i$, von $i=1$ an,

$$
\text { (a.) } \quad 1-\left(\begin{array}{l}
i \\
1
\end{array}\right)+\left(\begin{array}{l}
i \\
2
\end{array}\right)-\left(\begin{array}{l}
i \\
3
\end{array}\right)+\cdots \pm\left(\begin{array}{c}
i \\
i
\end{array}\right)=0
$$

und ferner, wenn $m$ eine ganze positive Zahl bezeichnet,

$$
\text { (b.) } \quad\left(\begin{array}{c}
i \\
1
\end{array}\right) 1^{m}-\left(\begin{array}{l}
i \\
2
\end{array}\right) 2^{m}+\left(\begin{array}{l}
i \\
3
\end{array}\right) 3^{m}-\cdots \pm i^{m}=0,
$$

so oft $i>m$ ist. Vermöge dieser Gleichungen reducirt sich die erste Summe auf das Glied, das $i=1$ entspricht, und dieses auf $-\frac{1}{2} \log a$.

Die zweite Summe zerlegt sich in

$$
\begin{aligned}
\Sigma \frac{a}{i+1}\left[-\left(\begin{array}{c}
i \\
1
\end{array}\right) \log \right. & \left.\left(1+\frac{1}{a}\right)+\left(\begin{array}{c}
i \\
2
\end{array}\right) \log \left(1+\frac{2}{a}\right)-+\cdots\right] \\
& +\Sigma \frac{1}{i+1}\left[-\left(\begin{array}{c}
i \\
1
\end{array}\right) 1 \log \left(1+\frac{1}{a}\right)+\left(\begin{array}{l}
i \\
2
\end{array}\right) 2 \log \left(1+\frac{2}{a}\right)-+\cdots\right] .
\end{aligned}
$$

Journal für Mathematik Bd. LVII. Heft 3. 
270 Bauer, von den Gammafunctionen w. einer besonderen Art v. Producten.

Entwickelt man nun die einzelnen Logarithmen in Reihen nach Potenzen von $\frac{1}{a}$, wobei freilich die Convergenz verloren geht, so wird mit Berücksichtigung der Relation (b.) die erste dieser Summen

$$
=-\frac{1}{2}+\sum_{m=1}^{m=\infty} \frac{(-1)^{m}}{(m+1) a^{m}} \sum_{i=1}^{i=m+1} \frac{1}{i+1}\left[-\left(\begin{array}{c}
i \\
1
\end{array}\right) 1^{m+1}+\left(\begin{array}{c}
i \\
2
\end{array}\right) 2^{m+1}-\left(\begin{array}{c}
i \\
3
\end{array}\right) 3^{m+1}+-\cdots\right]
$$

und die zweite

$$
=\sum_{m=1}^{m=\infty} \frac{(-1)^{m-1}}{m a^{m}} \sum_{i=1}^{i=m+1} \frac{1}{i+1}\left[-\left(\begin{array}{l}
i \\
1
\end{array}\right) 1^{m+1}+\left(\begin{array}{c}
i \\
2
\end{array}\right) 2^{m+1}-\left(\begin{array}{l}
i \\
3
\end{array}\right) 3^{m+1}+-\cdots\right] .
$$

Fassen wir dies Alles zusammen und bemerken zugleich, dafs

ist, so kommt

$$
\log C=\log \sqrt{2 \pi e}=\log \sqrt{2 \pi}+\frac{1}{2}
$$

(22.) $\log \Gamma(a)=\log \sqrt{2 \pi}+\left(a-\frac{1}{2}\right) \log a-a+\sum_{m=1}^{m=\infty} \frac{(-1)^{m} E_{m}}{m(m+1)} \frac{1}{a^{m}}$,

wo

$$
\boldsymbol{E}_{m}=\sum_{i=1}^{i=m+1} \frac{1}{i+1}\left[\left(\begin{array}{c}
i \\
1
\end{array}\right) 1^{m+1}-\left(\begin{array}{c}
i \\
2
\end{array}\right) 2^{m+1}+\left(\begin{array}{l}
i \\
3
\end{array}\right) 3^{m+1}-+\cdots\right]
$$

gesetzt ist.

Vergleicht man diese Entwicklung mit der Stirlingschen Formel für $\log (1.2 .3 \ldots a)$, so sieht man erstens, dafs $\boldsymbol{E}_{m}=0$ sein mufs, so oft $m$ eine gerade Zahl ist. Bezeichnen wir ferner mit $\boldsymbol{B}_{1}, \boldsymbol{B}_{3}, \boldsymbol{B}_{5}, \ldots \boldsymbol{B}_{2 m-1}$ die $1^{\text {ste }}, 2^{\text {te }}, 3^{\text {te }}, \ldots m^{\text {te }}$ Bernoullische Zahl, so mufs $\boldsymbol{E}_{m}=(-1)^{\text {t(m+1) }} \boldsymbol{B}_{m}$ sein, wenn $m$ eine ungerade Zahl ist.

11. Andere, wie ich glaube, neue Relationen und independente Ausdrücke für die Bernoullischen Zahlen erhält man durch die Entwicklung von $\log \Gamma(a)$ aus den in Nr. 9 gegebenen Formeln. So findet man, dafs die Summen

$$
\begin{aligned}
& \sum_{i=1}^{i=m+1} \frac{1}{i+1}\left[\left(\begin{array}{c}
i \\
1
\end{array}\right) 1^{m+1}-\left(\begin{array}{c}
i \\
2
\end{array}\right) 2^{m+1}+\left(\begin{array}{c}
i \\
3
\end{array}\right) 3^{m+1}-+\cdots\right], \\
& i=m+1 \\
& \sum_{i=1}^{i} \frac{-1}{i(i+1)}\left[\left(\begin{array}{c}
i \\
1
\end{array}\right) 1^{m+1}-\left(\begin{array}{c}
i \\
2
\end{array}\right) 2^{m+1}+\left(\begin{array}{c}
i \\
3
\end{array}\right) 3^{m+1}-+\cdots\right], \\
& \left.\sum_{i=m}^{i=1} \frac{1.2}{i(i+1)(i+2)}\left[\begin{array}{c}
i+1 \\
1
\end{array}\right) 1^{m+1}-\left(\begin{array}{c}
i+1 \\
2
\end{array}\right) 2^{m+1}+\left(\begin{array}{c}
i+1 \\
3
\end{array}\right) 3^{m+1}-+\cdots\right]+m-\frac{1}{2}, \\
& \begin{array}{c}
i=m-1 \\
\sum_{i=1}^{i} \frac{-1.2 .3}{i(i+1)(i+2)(i+3)}\left[\left(\begin{array}{c}
i+2 \\
1
\end{array}\right) 1^{m+1}-\left(\begin{array}{c}
i+2 \\
2
\end{array}\right) 2^{m+1}+\left(\begin{array}{c}
i+2 \\
3
\end{array}\right) 3^{m+1}-+\cdots\right] \\
+\frac{13}{6}+m-2^{m}\left(\frac{8}{3}-m\right)
\end{array}
\end{aligned}
$$


Bauer, von den Gammufunctionen $u$. einer besonderen Art v. Producten. 271

u. a. $m$. sämmtlich $=0$ sind, wenn $m$ gerade, und

$$
=(-1)^{\frac{1}{2}(m+1)} \boldsymbol{B}_{m}, \quad \text { wenn } m \text { ungerade. }
$$

Diese Relationen lassen sich noch etwas einfacher ausdrücken. Setzen wir

$$
1^{m}-\left(\begin{array}{c}
i \\
1
\end{array}\right) 2^{m}+\left(\begin{array}{c}
i \\
2
\end{array}\right) 3^{m}-+\cdots \pm(i+1)^{m}=N_{i}^{(m)},
$$

eine Gröfse, welche vermöge der Relation (b.) auch $=0$ ist, wenn $i>m$, so lassen. sich obige Relationen auch auf folgende Art schreiben

$$
\left.\begin{array}{c}
\sum_{i=1}^{i=m+1} \frac{i}{i+1} N_{i-1}^{(m)} \\
\sum_{i=1}^{i=m+1} \frac{-1}{i+1} N_{i-1}^{(m)} \\
\sum_{i=1}^{i=m} \frac{1.2}{i(i+2)} N_{i}^{(m)}+m-\frac{1}{2} \\
\sum_{i=1}^{i=1} \frac{-1.2 .3}{i(i+1)(i+3)} N_{i+1}^{(m)}+\frac{13}{6}+m-2^{m}\left(\frac{8}{3}-m\right)
\end{array}\right\}=0, \text { wenn } m \text { gerade, }
$$

Aus der Verbindung dieser Formeln folgt sodann, dafs für jede ganze Zahl $m$

$$
\begin{aligned}
& \sum_{i=1}^{i=m+1} N_{i-1}^{(m)}=0, \\
& \sum_{i=1}^{i=m} \frac{1}{i} N_{i}^{(m)}=-m, \\
& \sum_{i=1}^{i=1} \frac{1}{i(i+1)} N_{i+1}^{(m)}=1+m \cdot 2^{m-1}-2^{m}
\end{aligned}
$$

u. a. dgl. Die hier gegebenen Ausdrücke für die Bernoullischen Zahlen sind für die Berechnung derselben weniger bequem als die von Laplace und Scherk aufgestellten; sie scheinen mir aber insofern bemerkenswerth, als sie mittelst der Gröfse $\boldsymbol{N}_{i}^{(m)}$ zusammengesetzt sind, welche in der Analyse sich öfter darbietet.

12. Was den Rest der semiconvergenten Reihe in (22.) betrifft, so möchte aus der Entwicklung in Nr. 10 nicht leicht ein einfacher Ausdruck fúr denselben zu ermitteln sein. Herr Lipschitz gab in der im Eingang erwähnten Abhandlung den genauen Rest der Reihe, wenn man dieselbe nach dem Gliede $(-1)^{n-1} \frac{B_{2 n-1}}{(2 n-1) 2 n} \frac{1}{a^{2 n-1}}$ abbricht, in der Form

$$
(-1)^{n} k_{n}=\frac{(-1)^{n} \cdot 2}{(2 \pi)^{2 n+2}} \int_{0}^{\infty} \sum_{i=1}^{i=\infty} \frac{y^{2 n} e^{-a y}}{i^{2 n}\left(i^{2}+\frac{y^{2}}{4 \pi^{2}}\right)} \partial y \text {. }
$$


272 Bauer, von den Gammafunctionen u. einer besonderen Art v. Producten.

Ich gelangte schon vor langer Zeit auf anderem Wege zu der einfacheren Form

$$
\text { (23.) } \boldsymbol{R}_{n}=\frac{-1}{\pi} \int_{0}^{\infty} \frac{\log \left(1-e^{-2 \pi a x}\right)}{1+x^{2}} x^{2 n} \partial x \text {, }
$$

welche sich übrigens auch sogleich aus der von Herrn Lipschitz gegebenen Form herleiten läfst. Es reicht hin in derselben $2 \pi$. ix an die Stelle von $y$ zu setzen, wodurch die Ausführung der Summation ermöglicht wird. Der Rest $\boldsymbol{R}_{n}$ ist, wie Herr Lipschitz schon bemerkte, kleiner als $\frac{\boldsymbol{B}_{2 n+1}}{(2 n+1)(2 n+2)} \frac{1}{a^{2 n+1}}$. Dies läfst sich auch leicht aus der Form (23.) des Restes nachweisen. Ist nämlich $n$ eine ganze Zahl, so ist

folglich

$$
\int_{0}^{\infty} e^{-s x} x^{2 n} \partial x=\frac{1.2 .3 \ldots 2 n}{s^{2 n+1}}
$$

$$
\sum_{s=1}^{s=\infty} s \int_{0}^{\infty} e^{-s x} x^{2 n} \partial x=1.2 .3 \ldots 2 n \sum \frac{1}{s^{2 n}}=\frac{1}{2}(2 \pi)^{2 n} \boldsymbol{B}_{2 n-1} .
$$

Zweimalige theilweise Integration aber giebt

$$
s \int_{0}^{\infty} e^{-s x} x^{2 n} \partial x=\frac{2 n(2 n-1)}{s} \int_{0}^{\infty} e^{-s x} x^{2 n-2} \partial x,
$$

und hierdurch geht die letzte Gleichung in folgende über

oder

$$
\sum_{s=1}^{s=\infty} \frac{1}{s} \int_{0}^{\infty} e^{-s x} x^{2 n-2} \partial x=\frac{1}{2} \frac{(2 \pi)^{2 n}}{2 n(2 n-1)} B_{2 n-1}
$$

$$
\int_{0}^{\infty} \log \left(1-e^{-x}\right) x^{2 n-2} \partial x=-\frac{1}{2} \frac{(2 \pi)^{2 n}}{2 n(2 n-1)} B_{2 n-1}
$$

und folglich auch

(24.) $-\frac{1}{\pi} \int_{0}^{\infty} \log \left(1-e^{-2 \pi \Delta x}\right) x^{2 n} \partial x=\frac{B_{2 n+1}}{(2 n+1)(2 n+2)} \frac{1}{a^{2 n+1}}$.

Dieses Integral ist aber offenbar gröfser als das in (23.), welches den numerischen Werth des Restes darstellt.

München, im Juli 1859. 